\title{
Star Formation Thresholds: The View from Inside the Galaxy
}

\author{
James Di Francesco \\ National Research Council of Canada, \\ Herzberg Programs in Astronomy \& Astrophysics, \\ 5071 West Saanich Road, Victoria, BC, Canada, V9E 2E7 \\ email: james.difrancesco@canada.ca
}

\begin{abstract}
We explore the relationship between the total gas surface density and star formation rate surface density, a.k.a., the "Kennicutt-Schmidt relation," in a Galactic context. Specifically, we probe the origins of thresholds in the behaviour of the K-S relation at $10 \mathrm{M}_{\odot} \mathrm{pc}^{-2}$ and $100-$ $200 M_{\odot} \mathrm{pc}^{-2}$ using images from the Herschel Hi-GAL and Gould Belt surveys. In both cases, pervasive filamentary structures are seen, possibly due to turbulent motions. The Hi-GAL image supports the view that at $\sim 10 \mathrm{M}_{\odot} \mathrm{pc}^{-2}$ gas becomes molecular, leading to the formation of clouds that harbour star formation. The GBS images suggest the 100-200 $\mathrm{M}_{\odot} \mathrm{pc}^{-2}$ threshold originates from the nature of filaments being stable until a critical column density of $\sim 160 \mathrm{M}_{\odot}$ $\mathrm{pc}^{-2}$ is reached. Therefore, the transition between non-star-forming and star-forming gas in clouds (and galaxies) may be set universally by the dynamical properties of filaments.
\end{abstract}

Keywords. stars: formation, ISM: clouds, ISM: structure, galaxies: ISM, submillimeter

\section{Introduction}

"Stars form out of dense cores in molecular clouds." This statement or variations thereof has been for decades a recurring first sentence in numerous publications about Galactic star formation. In general, "dense cores" have been defined as pockets of gas typically within larger molecular clouds with sizes of $\sim 0.1 \mathrm{pc}$, masses of $\sim 0.5 \mathrm{M}_{\odot}$, and densities of $>10^{4} \mathrm{~cm}^{-3}$ (see Di Francesco et al. 2007). Indeed, the strong connection between young stellar objects (YSOs) and dense gas goes back to the pioneering work of Myers \& Benson (1983), who found associations between $\mathrm{NH}_{3}(2,2)$ emission and the locations of "emission line stars" in Taurus-Auriga. Today, $\mathrm{NH}_{3}$ emission is used extensively to trace dense gas associated with cores in other Galactic star-forming regions (e.g., Friesen et al. 2009, Friesen et al. 2015, in prep). In addition, very strong correlations have been seen between the numbers of young stars produced and the amounts of gas seen at densities greater than $10^{4} \mathrm{~cm}^{-3}$ (i.e., extinctions of $A_{V}>8$ ) for nearby and Galactic clouds (see Johnstone, Di Francesco \& Kirk 2004; Wu et al. 2005; Lada, Lombardi \& Alves 2010; Sadavoy et al. 2014).

The clear association of dense gas with star formation has made the well-known "Kennicutt-Schmidt" (K-S) scaling relation hard for some Galactic astronomers (at least this one) to understand at face value. Indeed, the K-S relation is a tight power-law correlation between the surface density of the star formation rate $\left(\Sigma_{\mathrm{SFR}}\right)$ and the surface density of the total gas in galaxies (Kennicutt 1989; Kennicutt 1998). The total gas surface density is defined as the sum of the atomic and molecular hydrogen surface densities, $\Sigma_{\mathrm{HI}+\mathrm{H}_{2}}$, with no overt connection to line-of-sight amounts of dense gas. For reference, the density of a cold neutral HI cloud is $\sim 10 \mathrm{~cm}^{-3}$ and that of a molecular cloud is $\sim 10^{1.5-2}$ $\mathrm{cm}^{-3}$, not the $\sim 10^{4} \mathrm{~cm}^{-3}$ densities (or higher) of dense cores. Nevertheless, the origin 


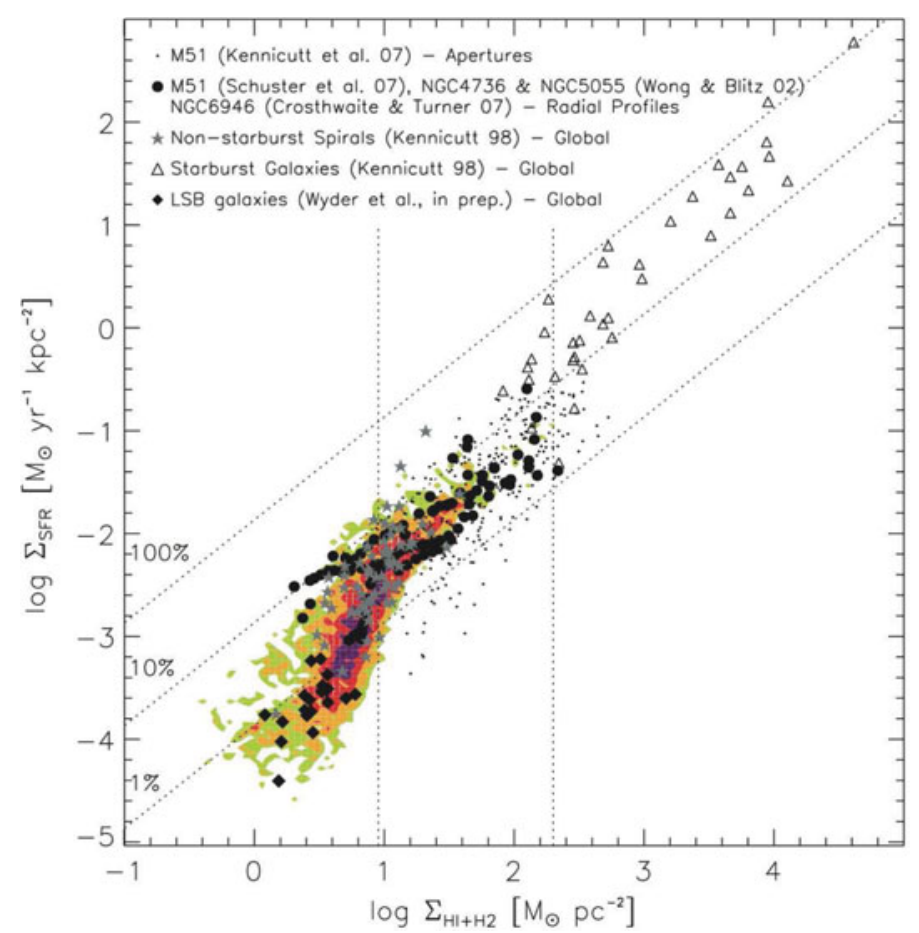

Figure 1. The relationship between total gas surface density $\left(\Sigma_{\mathrm{HI}+\mathrm{H}_{2}}\right)$ and star formation rate surface density $\left(\Sigma_{\mathrm{SFR}}\right)$ (a.k.a. the "Kennicutt-Schmidt relation") over a variety of galaxy types from Bigiel et al. (2008). The colour contours are from galaxies from their paper. Small and large black dots, stars, triangles, and black diamonds are other galaxy populations. Notably, the K-S relation is tested here on sub-kpc scales using resolved observations of several galaxies. The horizontal dashed lines indicate the two "thresholds," one at $\sim 10 \mathrm{M}_{\odot} \mathrm{pc}^{-2}$ and another at $100-200 \mathrm{M}_{\odot} \mathrm{pc}^{-2}$ where the behaviour of the relation changes. The diagonal dashed lines show lines of constant star formation efficiency, indicating the level needed to consume $1 \%$ (lower) to $10 \%$ (middle) to $100 \%$ (upper) of available gas in $10^{8}$ years. See Bigiel et al. (2008) for assumptions regarding IMF, CO line ratio, $\mathrm{X}$-factors and galaxy inclinations.

and interpretation of the K-S law have been matters of great debate among extragalactic observers and theorists for almost two decades (e.g., see Kennicutt \& Evans 2012).

\section{The Kennicutt-Schmidt Relation and its Thresholds}

Figure 1 shows a determination of the K-S relation by Bigiel et al. (2008) that includes observational data from low surface brightness (LSB) galaxies, non-starburst spirals, starburst galaxies, and resolved observations of the nearby galaxies M51, NGC 4736, NGC 5055, and NGC 6946. The achievement here was that the K-S relation was explored on sub-kpc scales. Though a degree of overall correlation is still seen, the power-law behaviour of the K-S relation is seen to vary somewhat over total gas surface density. At lower values, i.e., $\Sigma_{\mathrm{HI}+\mathrm{H}_{2}}<10 \mathrm{M}_{\odot} \mathrm{pc}^{-2}$, the relation is broad and relatively steep, but at higher values, the relation is shallower. Furthermore, somewhere around $\Sigma_{\mathrm{HI}+\mathrm{H}_{2}}$ $\sim 100-200 \mathrm{M}_{\odot} \mathrm{pc}^{-2}$, the relation becomes steeper. The two values of total gas surface density appear to correspond to thresholds for star formation production in galaxies, at least when averaged on sub-kpc scales along the line of sight.

The spatial averaging that is inescapable when observing external galaxies indeed makes interpreting the K-S relation tricky. Examining where star-forming Galactic clouds 


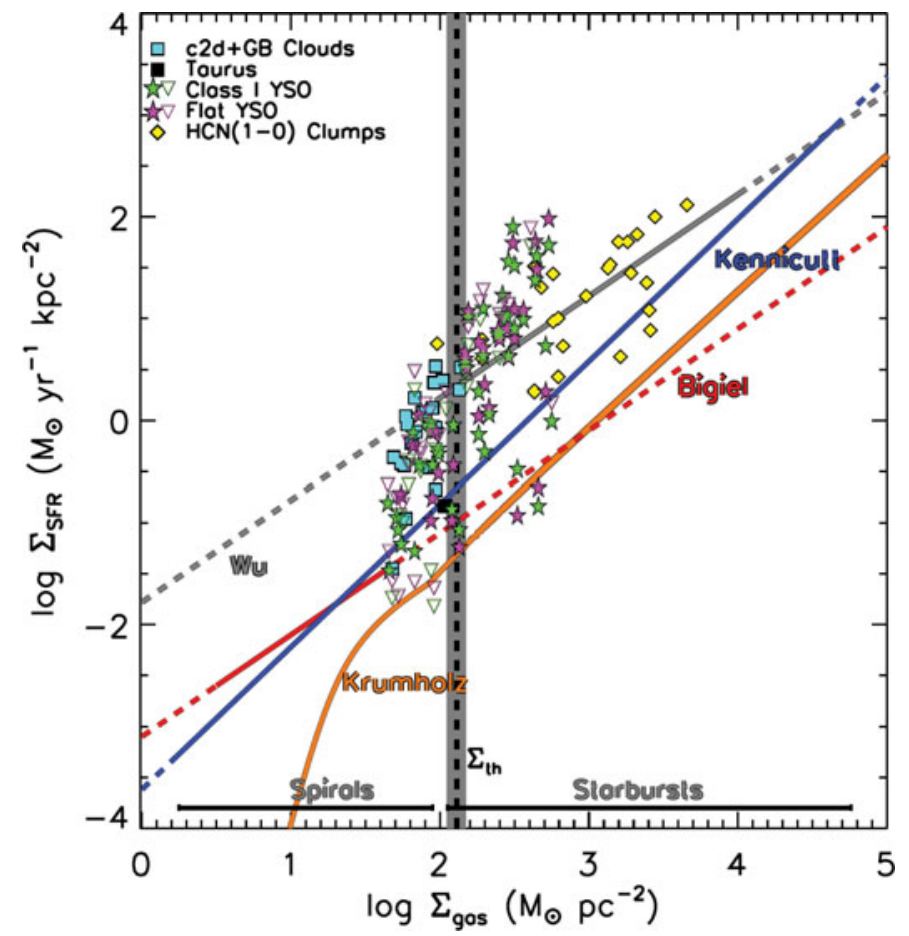

Figure 2. The relationship between gas surface density $\left(\Sigma_{\text {gas }}\right)$ and SFR surface density $\left(\Sigma_{S F R}\right)$ for Galactic objects from Heiderman et al. (2010). Squares indicate nearby clouds whose YSO populations were enumerated by Spitzer, stars indicate protostellar cores and diamonds indicate "massive" clumps. The extragalactic K-S scaling relations found by Kennicutt (1998) and Bigiel et al. are indicated by blue and red lines. The threshold in gas surface density where the relationship changes is marked by a vertical grey bar and black dashed line. Note how the Galactic objects have $\Sigma_{S F R}$ values much larger than those expected from the extragalactic scaling relations.

lie on a K-S relation diagram is instructive. For example, Figure 2 shows a determination of the K-S relation for Galactic clouds by Heiderman et al. (2010). Using Spitzer data to determine the actual YSO content of nearby clouds, these authors found that such clouds have $\Sigma_{\text {SFR }}$ values about an order of magnitude above those expected from Figure 1 for given total (molecular) gas surface densities. For individual protostellar cores, the discrepancy was found to be even larger. If such clouds and cores were placed in external galaxies, however, the observed total gas surface densities would be much lower, as they would be included in a spatial element with other non-star-forming HI and molecular gas. In addition, their YSOs would be hard to detect (if at all). Hence, a different conclusion about the relation between $\Sigma_{\mathrm{SFR}}$ and $\Sigma_{\mathrm{HI}+\mathrm{H}_{2}}$ would result. As an aside, it is worth noting that when Heiderman et al. added dense Galactic clumps traced by HCN (1-0) into the mix, they found the trend in $\Sigma_{\mathrm{SFR}}$ with $\Sigma_{\mathrm{HI}+\mathrm{H}_{2}}$ changing at $129 \pm 14 \mathrm{M}_{\odot} \mathrm{pc}^{-2}$, a value similar to the second threshold seen by Bigiel et al.

The transitions seen in Figure 1 can be understood as depending on what clouds at which phase are dominant within a spatial element of a galaxy. A single spatial element may encompass multiple phases of the ISM, including several neutral HI and molecular clouds. At lower $\Sigma_{\mathrm{HI}+\mathrm{H}_{2}}$ values, the gas on average is $<10 \mathrm{M}_{\odot} \mathrm{pc}^{-2}$, a value corresponding (within a factor of 2) to a line-of-sight extinction $A_{V} \sim 1$. Below that extinction, selfshielding of the ISRF by $\mathrm{H}_{2}$ becomes ineffective, and molecular gas cannot be sustained 

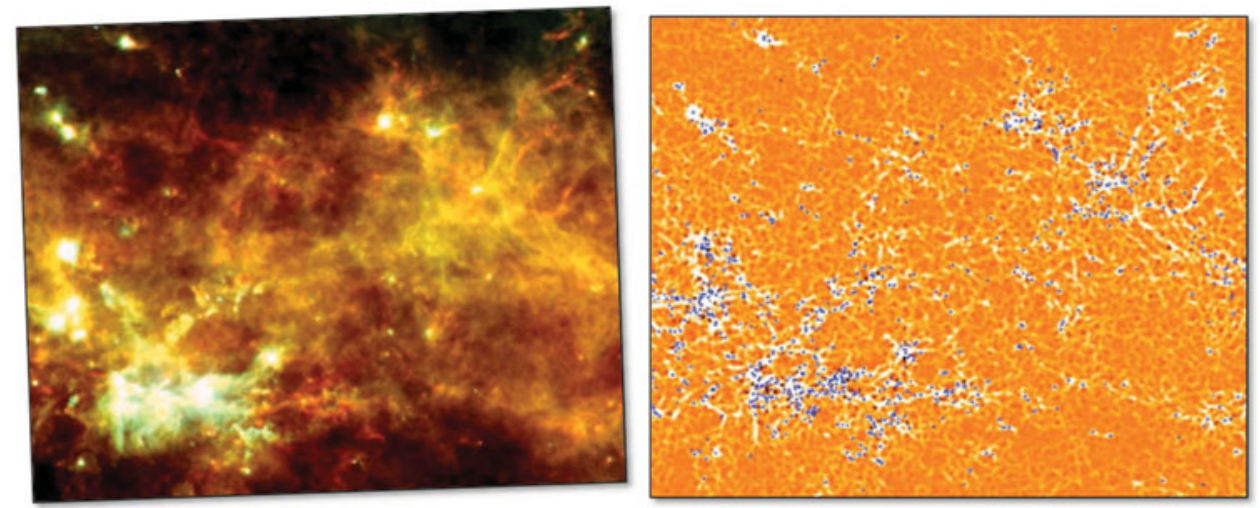

Figure 3. (left): Three-colour image composed of $70 \mu \mathrm{m}$ (blue), $160 \mu \mathrm{m}$ (green) and $350 \mu \mathrm{m}$ (red) of the Hi-GAL field around $l=59^{\circ}$ from Molinari et al. (2010). The left panel is tilted slightly to correspond spatially with features in the right panel. (right): A multidirectional second-derivative image of the same field as in the left from $250 \mu \mathrm{m}$ data, also from Molinari et al. (2010). White denotes locations of maximal emission, which to first order trace locations of peak column density. Blue circles represent compact sources detected in the $250 \mu \mathrm{m}$ image. This representation clearly reveals a web-like structure in the ISM due to filaments. The compact structures, likely clouds and dense cores within them, are found preferentially where $A_{V}>1$.

(see Krumholz et al. 2009). Hence, the low $\Sigma_{\mathrm{HI}+\mathrm{H}_{2}}$ regions of galaxies have on average low amounts of molecular gas. Star formation, however, is still found in such elements because there can be within them at least some molecular gas with pockets of dense gas that form stars. Such gas is relatively rare, however, and so star formation may be effectively "stochastic" with no true relation to total gas density (Kennicutt \& Evans 2012). Above $\Sigma_{\mathrm{HI}+\mathrm{H}_{2}} \approx 10 \mathrm{M}_{\odot} \mathrm{pc}^{-2}$, however, spatial elements include on average gas that exceeds the threshold for molecular persistence. In such clouds, a more regular trend is seen between $\Sigma_{\mathrm{HI}+\mathrm{H}_{2}}$ and $\Sigma_{\mathrm{SFR}}$, indicating some percentage of gas dense enough to form stars is commonly present. The dashed lines in Figure 1 suggest star formation efficiencies in this regime as being 1-10\%, and the large scatter suggests molecular clouds do not have a common percentage of gas within them that is dense enough to form stars. Around $\Sigma_{\mathrm{HI}+\mathrm{H}_{2}} \approx 100-200 \mathrm{M}_{\odot} \mathrm{pc}^{-2}$, a second transition is seen where star formation efficiencies jump to $10-100 \%$. At these values, the spatial elements (here, entire starburst galaxies) now encompass on average dense gas with extinctions $A_{V}>8$ that forms stars, and the SFR surface density rises accordingly.

\section{Herschel Continuum Observations of Galactic Clouds}

Though the nature behind the first transition is fairly well understood, the same cannot be said about the second. What makes gas where extinctions exceed $A_{V}>8$ so efficient at forming stars? Recently, continuum observations of Galactic clouds by ESA's Herschel Space Observatory have provided important clues about this threshold. Herschel was a 3.5-m diameter submillimeter space observatory situated at the Sun-Earth L2 point that operated from 2009 to 2013. Two of its instruments, PACS (Poglitsch et al. 2010 and SPIRE (Griffin et al. 2010) had photometric modes that allowed simultaneous mapping of emission from dust at $70 \mu \mathrm{m}$ or $100 \mu \mathrm{m}$ and $160 \mu \mathrm{m}$ (PACS) and $250 \mu \mathrm{m}$, $350 \mu \mathrm{m}$, and $500 \mu \mathrm{m}$ (SPIRE). These wavelengths are unobservable from the ground due to atmospheric absorption, and Herschel allowed wide-field mapping of cold Galactic clouds (among other targets) at unprecedented sensitivity and dynamic range. In partic- 
ular, Herschel detected from clouds faint large-scale emission that was previously unseen by ground-based observatories utilizing atmospheric windows (e.g., at $850 \mu \mathrm{m}$ ) due to the necessary removal from such data of large-scale emission from the atmosphere itself. In the following, we discuss some results from a few Herschel projects related to star formation thresholds.

Figure 3 (left) shows a multi-color image obtained with Herschel of the $l=59^{\circ}$ field for the large Hi-GAL survey of the Galactic Plane by Molinari et al. (2010). The $\sim 3.5 \mathrm{deg}^{2}$ field exhibits considerable complexity with emission seen at all scales and the brighter knots denote star-forming cloud complexes. Notably, colour in this image broadly reflects temperature, with redder locations being dominated by $500 \mu \mathrm{m}$ emission and thus cold. Figure 3 (right) is the same field as Figure 3 (left) but shows the second derivatives of the $250 \mu \mathrm{m}$ emission, done to reveal spatial relationships between locations of locally bright emission. Here, white shows locations of maximal emission, and these appear connected in a web-like arrangement of filamentary structures across the entire field. Blue circles indicate the locations of compact sources at $250 \mu \mathrm{m}$, and a very close correspondence is seen between the locations of such objects and the filamentary structure. Indeed, the compact sources, here molecular clouds or dense structures within them, are seen only at column densities equivalent to extinctions $A_{V}>1$. This correspondence demonstrates the close connection between the persistence of molecular gas and extinctions of $A_{V}$ $>1$, i.e., the first threshold in Figure 1. Crucially, however, Figure 3 (right) suggests a filamentary origin of the molecular clouds, possibly one shaped by dynamical (e.g., turbulent) flows where atomic gas is shaped into filaments within which only the densest locations exceed $A_{V} \sim 1$ and become molecular.

Closer to home, Herschel data show nearby molecular clouds have ubiquitous internal filamentary structures. With a more-detailed look at such clouds than generally possible with Hi-GAL, the conditions for star formation can be better seen. For example, Figure 4 shows mid-scale spatial components of column densities derived from SPIRE and PACS data of the nearby Aquila Rift (left) and Polaris Flare (right) molecular clouds by the Herschel Gould Belt Survey (André et al. 2010; Ward-Thompson et al. 2010; Könyves et al. 2010). Here, the column densities from compact and diffuse structures have been suppressed via a curvelet decomposition. The Aquila Rift is an active star-forming cloud containing the Serpens South and W40 clusters and the Polaris Flare is a relatively low column density cloud with no known star formation. Regardless of star-forming activity, however, Figure 4 shows that filamentary structures pervade both clouds. Indeed, the presence of filaments in both suggests an origin for the structure that is independent of star formation itself, and, as with the larger-scale filaments seen in Figure 3, one perhaps related to turbulence. Figure 5 shows a histogram of deconvolved filament widths determined from non-decomposed column density images of eight nearby clouds, including Aquila and Polaris (see Arzoumanian et al. 2011; André et al. 2014). Interestingly, the filamentary structures in these clouds have a narrow range of widths around $\sim 0.1 \mathrm{pc}$, despite a factor of 4 range in distance. The $0.1 \mathrm{pc}$ scale is where thermal and turbulent velocities are equivalent (see Larson 1981), again suggesting a turbulent origin.

Figure 4 (left) also shows a close association of gravitationally bound prestellar cores and known Class 0 objects with the densest filaments, i.e., those exceeding an extinction $A_{V} \sim 8$, in Aquila. Quantitatively, $\sim 75 \%$ of prestellar cores are found above the equivalent column density of $130 \mathrm{M}_{\odot} \mathrm{pc}^{-2}$. Clearly, core formation is more efficient within sufficiently dense filaments. Indeed, the filamentary morphology so prevalent within clouds may provide the key to understanding the initial conditions for star formation, and perhaps also the key to the second threshold of Figure 1. Representing filaments 

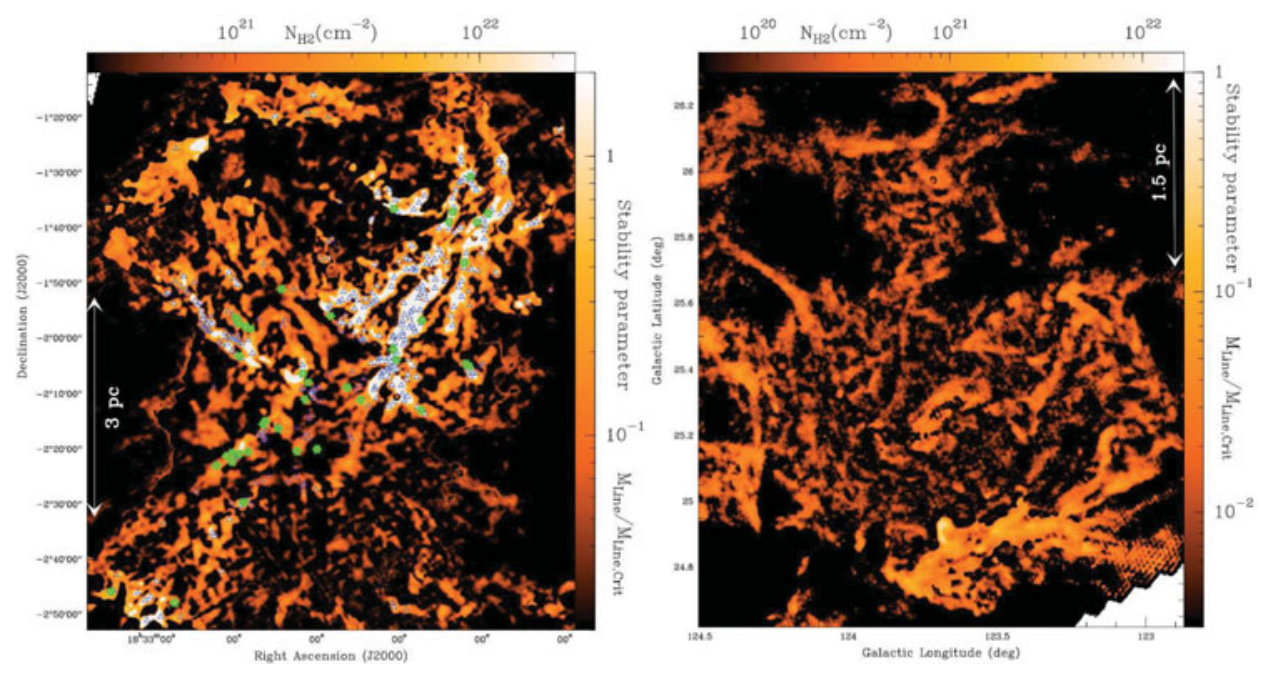

Figure 4. Column density maps of subfields of the Aquila Rift (left and the Polaris Flare (right) derived from SPIRE and PACS data obtained by the Herschel Gould Belt Survey from André et al. (2010). The maps have been spatially decomposed to highlight mid-scale spatial structures in these clouds. Large-scale, diffuse structure and small-scale, compact structures have been effectively suppressed. Linear filamentary features are seen to pervade both clouds. The colour scale is different for each image. On the left, the column densities for Aquila range between $\sim 2 \times 10^{20} \mathrm{~cm}^{-2}$ (black) and $\sim 2 \times 10^{22} \mathrm{~cm}^{-2}$ (white). On the right, the range for Polaris is $\sim 7 \times 10^{19} \mathrm{~cm}^{-2}$ to $\sim 10^{22} \mathrm{~cm}^{-2}$. Assuming a common filamentary width of $0.1 \mathrm{pc}$, the colour scale also reflects the local lines per unit length, with the ratio of $\mathrm{M}_{\text {line }} / \mathrm{M}_{\text {line,crit }}$ extending from $\sim 0.02$ to $\sim 2$ on the left and from $\sim 0.002$ to 1 on the right. Blue triangles denote prestellar cores and green stars denote Class 0 young stellar objects. Note the clear spatial correspondence between such objects and filaments of line per unit length above the critical value. Of the two clouds, only in Aquila are such high column densities reached, and consequently star formation is ongoing.

theoretically as isothermal cylinders, the stability of such structures to fragmentation can be understood as depending simply on a critical mass per unit length defined as $M_{\text {line }}=2 c_{s}^{2} / \mathrm{G}$ (Inutsuka \& Miyama 1997; see also Ostriker 1964). For typical temperatures of $10 \mathrm{~K}$ in a molecular cloud, this critical value is $16 \mathrm{M}_{\odot} \mathrm{pc}^{-1}$. Above this value, perturbed filaments will segment, i.e., fragment locally along their long axes, into cores rather than globally collapse (Pon et al. 2011). The colour scale of Figure 4 has been fixed so that filaments whose $M_{\text {line }}$ values exceed the critical value are white, illustrating the close connection between star formation activity and such filaments.

Given the seemingly common filament width of $\sim 0.1$ pc (see Figure 5), the critical $\mathrm{M}_{\text {line }}$ translates to a critical column density of $\sim 160 \mathrm{M}_{\odot} \mathrm{pc}^{-2}$ which is approximately equivalent to an extinction $A_{V} \sim 8$. Hence, we can understand the higher efficiency of star formation in filaments as resulting from the ability of such structures to fragment effectively into cores. Real filaments, however, are not strictly isothermal cylinders and exhibit small $(<5 \mathrm{~K})$ temperature dips in their interiors (see Palmeirim et al. 2013) and they likely have internal turbulent velocities on par with their thermal velocities. Moreover, they exhibit a small but finite distribution of widths around $0.1 \mathrm{pc}$ (see Figure 5 ), and may be inclined relative to the plane of sky. For these reasons, the observed "threshold" for core/star formation is not necessarily sharp at $160 \mathrm{M}_{\odot} \mathrm{pc}^{-2}$ (see André et al. 2014). 


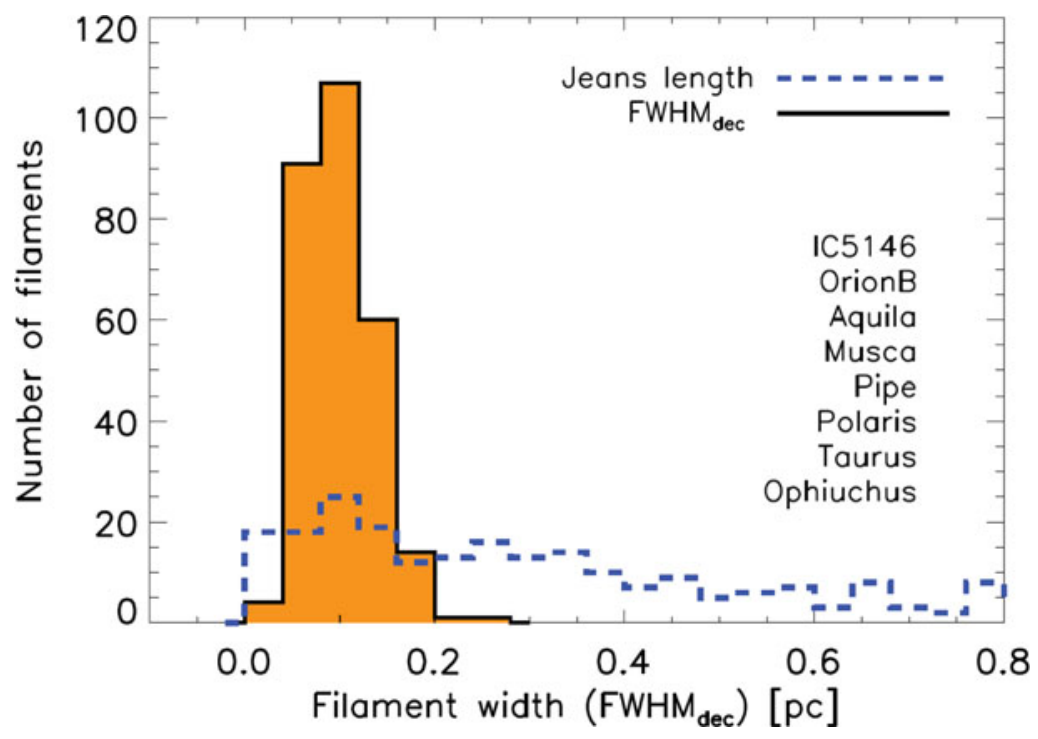

Figure 5. Histogram of deconvolved FWHMs of 278 filaments recovered in eight molecular clouds by Herschel GBS observations from André et al. (2014). The widths were determined from Gaussian fits to the filament profiles obtained from non-decomposed (all-scale) column density maps. The distribution is peaked at $\sim 0.1 \mathrm{pc}$ with a width of $\sim 0.04 \mathrm{pc}$. For comparison, the much wider distribution of Jeans lengths corresponding to the central column densities of the filaments is also shown as the blue-dashed histogram.

\section{A Universal Threshold for Star Formation?}

The similarity between the critical column densities seen for filaments in nearby clouds and the second threshold of Figure 1 for galaxies strongly suggests that the filamentary morphology and notably its critical requirement for instability are responsible physically and universally for the transition between diffuse, non-star-forming molecular gas and dense, star-forming molecular gas. Indeed, filaments may provide an effective means for gas to condense within clouds to produce through fragmentation a mass distribution of cores that is similar in shape to the stellar IMF, as seen directly in Aquila by Könyves et al. (2010) (see also Könyves et al. 2015). In Aquila, the peak of the core mass function is $0.6 \mathrm{M}_{\odot}$ which is roughly equal to the locally critical Bonnor-Ebert mass of $0.5 \mathrm{M}_{\odot}$. Moreover, a segment of $0.1 \mathrm{pc}$ length in a critical isothermal filament has a typical mass of $1.6 \mathrm{M}_{\odot} \approx 3 \times \mathrm{M}_{B E}$, indicating that the typical critical filament is locally unstable. Hence, we may understand the peak of the clump mass function, and perhaps the IMF, as indeed due to thermal fragmentation (Larson 1985). Furthermore, an recent analysis of 1-D fluctuations along 80 filaments by Roy et al. (2015) revealed a mean power spectrum slope of -1.6 , similar to the slope of -1.5 from Kolmogorov turbulence. Inutsuka (2001) previously showed such fluctuations could evolve naturally towards a core mass function with a observed Salpeter-like slope $(\alpha \approx-2.35)$ at higher masses.

Though highly suggestive, further work is needed to test the robustness of the connection between the critical column density of filamentary fragmentation and the observed threshold to higher star forming efficiencies in Figure 1. Notably, the Herschel GBS has concentrated primarily on local clouds that do not produce the kinds of high-mass stars used to determine SFRs in distant galaxies. It would be highly instructive to explore the relationship between filamentary structures in giant molecular clouds and high-mass star formation. Preliminary work of this kind has begun using data of giant molecular clouds within $3 \mathrm{kpc}$ from the Herschel OB Young Star (HOBYS) survey (Hill et al. 2011; 
Schneider et al. 2012). Furthermore, studies of the dynamics of filaments using robust kinematic tracers are also needed, to determine how such structures are evolving. Preliminary work also has begun in this direction. (Schneider et al. 2010; Arzoumanian et al. 2014; Friesen et al. 2015, in prep).

\section{Acknowledgement}

The author thanks Philippe André, Derek Ward-Thompson, Shu-Ichiro Inutsuka, Ralph Pudritz, and Jaime Pineda for the stimulating collaboration on which this paper is based. The author also thanks the Hi-GAL, GBS, and HOBYS Key Programme teams.

\section{References}

André, Ph., Di Francesco, J., Ward-Thompson, D., Inutsuka, S.-I., Pudritz, R., \& Pineda, J. 2014, in: H. Beuther, R. Klessen, P. Dullemond, \& Th. Henning (eds.), Protostars and Planets VI (Arizona: Tucson), p. 27

André, Ph., et al. 2010, A\& A, 518, L102

Arzoumanian, D., et al. 2013, A\&A, 553, A119

Arzoumanian, D., et al. 2011, A\&A, 529, L6

Bigiel, F., Leroy, A., Walter, F., Brinks, E., de Blok, W. J. G., Madore, B., \& Thornley, M. 2008, AJ, 136, 2846

Di Francesco, J., Evans, N. J., II, Caselli, P., Myers, P. C., Shirley, Y., Aikawa, Y., \& Tafalla, M. 2007, in B. Reipurth, D. Jewitt, \& K. Keil (eds.), Protostars and Planets V (Arizona: Tucson), p. 17

Friesen, R. K., Di Francesco, J., Shirley, Y., \& Myers, P. C. 2009, ApJ, 697, 1457

Griffin, M., et al. 2010, A\&A, 518, L3

Heiderman, A., Evans, N. J., II, Allen, L. E., Huard, T., \& Heyer, M. 2010, ApJ, 723, 1019

Hill, T., et al. 2011, A\& $A, 533$, A94

Inutsuka, S.-I. 2001, ApJ, 559, L149

Inutsuka, S.-I. \& Miyama, S. 1997, ApJ, 480, 681

Johnstone, D., Di Francesco, J, \& Kirk, H. M. 2004 ApJ, 611, L45

Kennicutt, R. 1998, ApJ, 498, 541

Kennicutt, R. 1989, ApJ, 344, 685

Kennicutt, R. \& Evans, N. J., II 2012, ARAA, 50, 531

Krumholz, M. R., McKee, C. F., \& Tumlinson, J. 2009 ApJ, 693, 216

Könyves, V., et al. 2015, $A \mathscr{E} A$, in press

Könyves, V., et al. 2010, A\& $A, 518$, L106

Lada, C. J., Lombardi, M., \& Alves, J. 2010, ApJ, 724, 687

Larson, R. B. $1985 M N R A S, 214,379$

Larson, R. B. 1981, MNRAS, 194, 809

Molinari, S., et al. 2010, A\& $A, 518, \mathrm{~L} 100$

Myers, P. C. \& Benson, P. 1983, ApJ, 266, 309

Ostriker, J. 1964, ApJ, 140, 1056

Palmeirim, P., et al. 2013, A\&A, 550, A38

Poglitsch, A., et al. 2010, A\& A, 518, L2

Pon, A., Johnstone, D., \& Heitsch, F. 2011, ApJ, 740, 88

Roy, A., et al. 2015, A\&\&A, in press

Sadavoy, S., et al. 2014, ApJ, 787, L18

Schneider, N., et al. 2012, A\& A, 540, L11

Schneider, N., et al. 2010, A\& A, 520, A49

Ward-Thompson, D., et al. 2010, A\&SA, 518, L92

Wu, J., Evans, N. J., II, Gao, Yu., Solomon, P. M., Shirley, Y. L., \& Vanden Bout, P. A. 2005, ApJ, 635, L173 